\title{
Research Directions in the Area of USN (Ubiquitous Sensor Network) Towards Practical UE (Ubiquitous Environments)
}

\author{
Young Yong Kim \\ School of Electrical and Electronic Engineering \\ Yonsei University \\ 120-742 Seoul, Korea \\ y2k@yonsei.ac.kr
}

\begin{abstract}
Ubiquitous" has been the key buzzword in the research community as well as in everyday life these days. In Korea, ubiquitous become decorative words for many new products, which has not necessarily enough link to the world of ubiquitous. In research field, many public, private sectors research activities decorated with the word of "ubiquitous" has drawn so much attention, but still, little examples are shown which truly implements the ideals of "Ubiquitous". In this paper, we review the research activities under the flag of "ubiquitous", and then present possible research directions for proper research directions which can prompt practical incorporation of ubiquitous concept in the near future.
\end{abstract}

\section{Introduction}

Recently, the word "ubiquitous" becomes truly ubiquitous in every area of our society from research community to TV commercials. Although ubiquitous technology grows faster than the clarification of the word itself, we paid less attention to the practical ubiquitous environments. Up to date, ubiquitous gadget exists somehow not really ubiquitous way, and the word "ubiquitous" presents more advertising values than technological merits. In research communities, we have covered technological issues such as ad-hoc network, USN (Ubiquitous Sensor Network), as well as RFID related issues. However, how these technological issues can really resolve into practical Ubiquitous Environments posts interesting research challenge as well.

In this paper, we present some bridge between ongoing as well as future technological perspective in the areas of USN and practical UE (ubiquitous environments). We show some very interesting ubiquitous applications and analyze the building blocks of USN technologies by top-down approaches. We show the importance of business rationale as well as social issues plays tremendous roles in order to extract any meaningful commercial values from the technological research activities.

\section{2 "Ubiquitous" Revisited}

Since Mark Weiser envisioned the word "Ubiquitous Computing" for the first time in 1988, even before cellular phone or Internet are widely accepted among end users, 
"ubiquitous" becomes such a buzz word recently that it is widely used even in TV commercials these days. According to his first definition, "Ubiquitous Computing" means that invisible everywhere computing that does not live on a personal device of any sort, but is in the woodwork everywhere. [1] Although, Mark Weiser was visionary in devising the concept for the first time, we should note that there has been tremendous development in the computing devices as well as networking technology. In communication technologies, we have noticed such breakthrough technology as Internet, and a variety of wireless communication revolutions ranging from cellular phones to near field communication.

With these in minds, we may need to revise what is current consensus about the word, "ubiquitous" in research communities as well as common sense in everyday life, in the age when ordinary people are repeatedly exposed to the word every day. Recently, even socialists pay attention to the word ubiquitous and they explain the impacts of technological development on our society with 3 space notions. The first space is called "R-space", where "R" stands for "Real". In this space, everyday interactions take places by direct human interactions. The second space is called "Espace", where E stands for "Electronic". Here, people interacts with each others in "cyber space" constructed on the web-space rather than in brick-and-mortar way. In E-space, people interacted with each others by telecommunications network rather than direct encounter. The third space is called "U-space" where U stands for "Ubiquitous". In U-space, R-space and U-space are mixed together to become U-space. In U-space, reality and cyber space are co-existent in everyday life due to "Invisible", "Ubiquitous" computing, communication devices everywhere. In U-space, R-space moves toward to E-space thanks to ubiquitous networking devices everywhere, while E-space approaches R-space via such technologies as augmented reality. Although definition of U-space $=\mathrm{E}$-space $+\mathrm{R}$-space is easy to say, it is not so clear to imagine concrete ideas of what services are original in U-space. We will consider many practical examples later.

"Ubiquitous" comes in different favors in different countries. In United States, ubiquitous computing and invisible computing are popular expressions when it comes to something related to U-space. In USA, ubiquitous and invisible are keywords for related ongoing or completed research projects as well.

In Europe, pervasive computing or ambient computing are more popular word for anything "U". Pervasive reiterate the ubiquity of U-space. Here again, these two words are popular keywords for research project names.

In Japan, "Ubiquitous Network" or UN in short is popular terminology, where TRON project is single dominant related project, which emphasize implementation of small devices for ubiquitous environments.

In Korea, Ubiquitous Sensor Network (USN) is the most popular terminology in government, research activities, while Ubiquitous word itself plays its role in many advertisement such as "ubiquitous apartment", "ubiquitous design", etc. The acronym, USN has been created by Korea Ministry of Information and Communication (MIC), and selected as one of the 3 infra structures, which is key in Korean MIC information technology 8-3-9 strategy. (The other 2 infra structures are BCN (Broadband Convergence Network) and IPv6). In Korea, USN is used in two meanings, broad sense as well as narrow sense. In broad sense, USN is equivalent to the notion of ubiquitous computing or pervasive networking, but emphasis on sensor devices. In narrow sense, 
we use RFID/USN where RFID means infrastructures based on RF (radio frequency) ID(Identification) NFC(Near Field Communication) and USN means Sensor Network Infrastructure based on sensor node. Sensor node means small devices which is composed of sensing devices, CPU, and communication devices. In the consequence, USN in narrow sense means network of sensor node or sensor network (SN) which is more frequently used in other countries or research activities. Therefore, USN in wide sense becomes infrastructure based on RFID as well as sensor network.

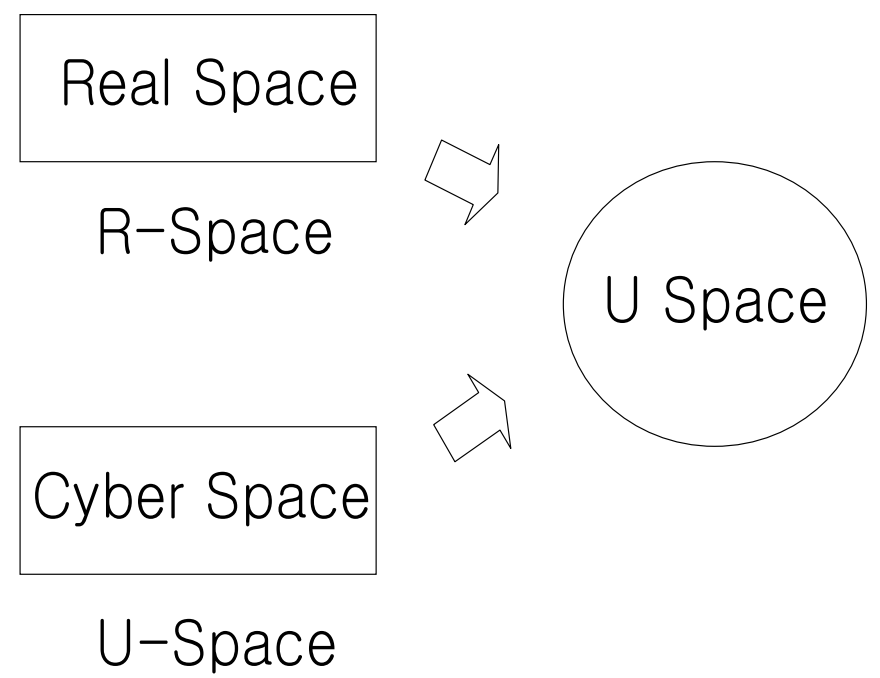

Fig. 1. U-Space

As we discussed, ubiquitous comes in a variety of meanings in different countries as well as in different areas in these day. Although the original notion of ubiquitous computing suggested by Mark Weiser has been inherited to current ubiquitous environments, current consensus on "ubiquitous" pose different focus in different countries and in different context. Therefore, one must be careful that the meaning of ubiquitous is not unanimous in different areas/countries, and should be understood under proper context.

\section{Ubiquitous Business}

Since the inception of notion in 1988, many research projects had been conducted under name of ubiquitous, and tremendous amount of papers have been published under the title of ubiquitous. However, in business or real life, one still finds it very difficult any concrete examples, which is truly "Ubiquitous" as envisioned by Mark Weiser. One of the most popular example of ubiquitous computing might be PDA(Personal Data Assistant) or any other very small hand held devices, which is so small that people can carry 
around easily. Although handheld devices are important stepping stones toward truly ubiquitous environments, small device alone cannot make the dream of Ubiquitous Environments (UE) completely. In addition, many ubiquitous oriented services including U-health, U-learning, U-city, or U-governments draws so much attention these days, but still what makes these services really ubiquitous is rather unclear. In this section, we try to clarify what is really ubiquitous business and how we can accelerate the progress from ubiquitous researches into ubiquitous business.

In different perspective, ubiquitous business in these days invites players from so many areas such as engineering, legal, social, economics, and business. If we call these as horizontal axes, vertical axis might be business model such as entertainment and gaming, tourism, pervasive retail, health care, logistics, and telemetric.

In previous section, we discuss the notion of R-space, E-space, and U-space. To better understand the ubiquitous business, we pervasive retail as prominent example. Although e-commerce has been replacing the traditional way of brick-andmortar retail business model, there certainly exists some areas where e-commerce cannot penetrate as originally expected. The areas where e-commerce (based on E-space) gaining more and more grounds against r-commerce (based on R-space) are generally selling some standardized goods such as computers, books, and audio compact discs. Common sense tells us that they are such standardized products that once you know the model number and maker, all you need to do is search the web retailer which is selling the items with the lowest price tag, as long as the service is not too coarse.

However, there are some items, which is not so successful in E-space. For examples, clothing and raw foods belong to that category. In general, people try to feel the textile, and try it on before they decide to buy. Therefore even when they buy it on the web in the long run, there should be fitting and try in R-space before e-commerce take place. Therefore, it is clear that commerce will benefit from combining $\mathrm{R}$-space and E-space together, namely, in U-space. U-commerce in U-space can benefit standardized items as well.

One possible scenario for U-commerce is that, a person visit some fashion brand show room (or department store) to try recent collections on. If she/he finds the items good to her/his taste, he scan RFID printed on the label of the clothes with the mobile RFDI reader, which is integrated into cellular phone. On the display of cellular phone, several online retailers' links shows up so that she/he can choose the one, namely with the lowest price tag. While she/he returns home, the selected item (with chosen size and chosen color) is already delivered. This is one example of u-commerce, which combine R-retailing as well as E-retailing. However, one question might be how showroom in R-space can benefit from this business model? The answer is that the profit of e-retailer is shared properly between the showroom in R-space and retailer in E-space. In this business model, the showroom owner possesses enough motivation to draw visitors by offering services and up to date stocks. In the consequence, with the aid of ubiquitous technologies u-commerce is far better advanced from of retailing by combining E-commerce and R-commerce in very efficient way. 


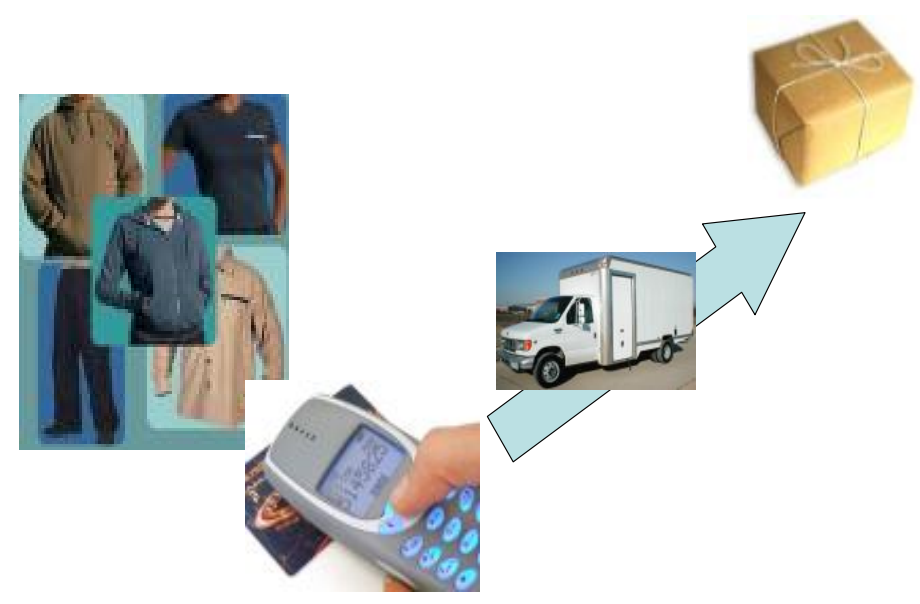

Fig. 2. U-commerce Scenario

Although RFID is considered the most visible bridge toward immediate ubiquitous business development, Sensor Network lags behind RFID in business model development with as-is status. Although, sensor node conforming to the original smart dust ideas in Berkeley[2], few business model have been proposed compared to RFID models. As long as author knows, one hop sensor network in a car is one prominent example of commercial sensor network available in the very near future.

\section{Future Research Directions}

So far tremendous amounts of papers have been published in the area of ubiquitous computing, sensor networking, and etc. If we count number of papers or amounts of research funds globally, sensor networking is among the top lists in recent IT research activities. Among sensor network research, substantial portions of the researches are focused on protocols design with the assumptions of increasingly peculiar situations. Although research communities made progress on its own, it is also true that the gap has been widened between u-business and ubiquitous researches.

Recently, growing amount of researches has been directed to the business model developments as well as developing practical building blocks of u-business. Although recent advancement in wireless communications and Internet paved the way to realization of Mark Weiser's dream, we still sees current situation far from invisible computing, especially in the area of sensor network. Therefore, any practical researches should be directed toward practical implementations as well, standing firmly on the ground.

\section{Conclusions}

In this paper, we revisited the notion of ubiquitous computing, and redefine practical ubiquitous environments with the concept of U-space which is leap forward 
combining real space together with the cyber space. We also propose some successful business model with the u-commerce in mind. Although we already enter into the business in u-space with the aid of RFID technology, we still have long way to go with the business development of Sensor Network.

The future research activities should be directed toward practical ubiquitous environments, and more attentions should be paid such key stepping stones as security related problems and business development tools.

\section{Acknowledgement}

This work was supported by HISP(Hybrid Intelligent Service Platform) project, funded by MOCIE(Ministry of Commerce, Industry and Energy), Korea.

\section{References}

1. Weiser, M.: The World is Not a Desktop. Interactions. (1994) 7-8

2. Warneker, B., Last, M., Leivowitz, B., Pister, K.S.J.: Smart Dust: Communicating with a Cubic-Millimeter Computer. Computer, Vol. 34. IEEE (2001) 44-51

3. Rajendran, V., Obraczka, K., and Garcia-Luna-Aceves, J.J: Energy-Efficient, Collision-Free Medium Access Control for Wireless Sensor Networks. Wireless Networks. Vol. 12. Springer, Netherland (2006) 63-78

4. Fano, A., Gershman A.: The Future of Business Services in the Age of Ubiquitous Computing. Communications of ACM, Vol. 45, ACM Press, New York, USA(2002) 83-87 